\title{
;

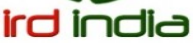 \\ Online Parameter Monitoring of Induction Motor Using Wireless Network
}

\author{
Geethi.P \& V.Saravanan \\ Vivekanandha College of Engineering For Women, Tiruchengode, Tamil Nadu, India
}

\begin{abstract}
Induction motor is used in majority of the industrial applications. The main reason for the usage of IM is its reliability and simplicity of operation. Most of electrical energy is utilized by induction motors. And thus it is essential to monitor the performance of the motor without changing its operation.
\end{abstract}

Here in this paper, introduces a new technique in which embedded system is integrated into the wireless network. During this technique, different sensors are connected with the motor and the values are extracted using a PIC microcontroller. It is then transmitted to the base station and at the base station a Graphical User Interface is given which give the user can interface with the system. The wireless protocol used is Zigbee.

Keywords: induction motor (IM), efficiency, on-site, energy audit.

\section{INTRODUCTION}

Embedded systems mainly contain processing cores that are either microcontrollers or digital signal processors (DSP). The key characteristic, however, is being dedicated to handle a particular task. Physically, embedded systems range from portable devices such as digital watches and MP3 players, to large stationary installations like traffic lights, factory controllers. Complexity varies from low, with a single microcontroller chip, to very high with multiple units, peripherals and networks mounted inside a large chassis or enclosure. The main applications of the embedded system include telecommunication system, Consumer electronics, and transportation systems from flight to automobiles, medical equipments and industrial automation.

Industrial automation is the process in which the manpower in the industry is reduced by automating the operations. Doing this not only manpower but also the time required for the operations can be improved. Industrial automation in coordination with the Mechatronics gives more efficient performance.
Mechatronics is the synergistic integration of sensors, actuators, signal conditioning, power electronics, decision and control algorithms, and computer hardware and software to manage complexity, uncertainty, and communication in engineered systems.

In an industrial environment, mechanical systems driven by electric motors are used in most production processes, accounting for more than two-thirds of industry electricity consumption. Motor-driven systems use nearly 70 percentage of the total electric energy consumed by industry. On average, these motors operate at no more than $60 \%$ of their rated load because of oversized installations or under loaded conditions, and thus at reduced efficiency which results in wasted energy.

About 90 percent of the total motor electricity consumption is done with ac. three phase induction motors in the power range from $0.75 \mathrm{~kW}$ to $750 \mathrm{~kW}$. In the industrial sector the motors use about 69percent of the total electricity and in territory sector it uses around 36 percent of electricity. Where the lighting circuits uses only 6 percent in industrial consumption and 30 mpercent in the territory consumption.

\section{LITERATURE SURVEY}

Significant efforts have been dedicated to induction machine efficiency monitoring during the last two decades and many techniques have been proposed. Thus, a brief description of the main techniques presented in the literature, as well as their advantages and disadvantages are presented in this section.

A non-intrusive and in-service motor efficiency estimation method was proposed in 2008, where the efficiency estimation was done using Air Gap torque method [1]. Only motor terminal quantities and nameplate details, with special considerations of motor condition monitoring requirements are required. Pre installed potential transformers and current transformers for protection purpose. But the system was valid only for motors with power $<20 \mathrm{hp}$. A low cost wireless sensor network for in-field operation monitoring of induction 
motor was proposed for high range motors [2]. Where a smart switch system was proposed. smart switch has a data logger that is used to monitor operation condition and automatically manages the motor winding connection mode. A non-intrusive efficiency estimation method was introduced without speed sensors [3]. But due to the lack of proper speed sensing drive, the system is said to be less efficient. Bacterial foraging algorithm along with a non-intrusive method is used for the efficiency estimation in [4]. But the system becomes lengthier in calculations. Use of genetic algorithm with equivalent circuit method was introduced in [5]. But the system is lengthy but easy to calculate.

\section{SYSTEM ANALYSIS}

\section{A. Existing system}

The system used in the latest existing system is embedded system integrated with wireless sensor network. A WSN is used here for making the calculations more reliable and non invasive.

At first the parameters are calculated using the embedded system circuit. Here used only two sensors. A Hall Effect voltage sensor and current sensor.

The current and voltage values are obtained from the sensors. Here Air Gap Torque method is used for the calculation of efficiency. That is first using the voltage and current values the Air Gap Torque is calculated. And this torque is recorded. From this the losses are subtracted from the air gap torque and the shaft torque is calculated.

Using this shaft torque we can calculate the mechanical efficiency. The efficiency can be obtained using formulas.

The AGT method proposed we can calculate the torque using the equation.

$T_{a g}=\frac{p \sqrt{3}}{6} \int i_{a}-i_{b} \int\left[v_{c a}+r\left(2 i_{a}+i_{b}\right)\right] d t+\left(2 i_{a}+i_{b}\right) \int\left[\left(v_{a b}-r\left(i_{a}-i_{b}\right)\right)\right]$

where

$p$ number of motor poles;

$i a$, ib motor line currents, in ampere;

$v c a, v a b$ motor power line voltages, in volt;

$r$ resistance of motor armature, in ohm.

Equation (1) can be applied using instantaneous and simultaneous acquisitions of $i a, i b, v c a, v a b$, and a measured value of $r$. It is valid both for motors connected in $Y$, with no connection to the neutral, or $\Delta$. Its integrals corresponding to the stator flux linkages. AGT equations has also been used in many works that use other types of motors. The torque on the shaft can be estimated by subtracting the losses occurring after the process of electromechanical energy conversion from AGT.

$$
\begin{aligned}
& T_{\text {shaft }}=T_{a g}-\frac{L_{m e c}}{\omega_{r}}-\frac{L_{R s l}}{\omega_{r}} \\
& P_{\text {in }}=i_{a} v_{a}+i_{b} v_{b}+i_{c} v_{c}=-v_{c a}\left(i_{a}+i_{b}\right)-v_{a b} i_{b} \\
& \eta=\frac{T_{\text {shaft }} \omega_{r}}{-v_{c a}\left(i_{a}+i_{b}\right)-v_{a b} i_{b}}
\end{aligned}
$$

The WSN used here is IEEE 802.15.4. Using this monitoring task can be done from a base station away from the motor.

The main drawback of this system is that, it requires more calculations and also here only mechanical efficiency is taken in to account. There is only one voltage and current sensors, so the speed and torque are not measuring directly, so there is a chance of error.

\section{A. $\quad$ Proposed system}

Analysis of induction motor is much essential to find out utilization index of a motor for better performance. When we analyze an efficiency of an induction motor we need to

acquire many parameters like voltage, current, KW, power factor, speed, torque from motors. All the above said parameters [7] must be acquired at fastest speed to present an instantaneous efficiency indication.

When we analyze these parameters we can easily identify whether the motor is suitable for particular operations or not, can be identified.

In our project, we are going to use all type of sensing system. For above said parameters all the sensors will be connected to signal conditioning circuits to convert signals suitable for interfacing with embedded controller. The state of art PIC microcontroller manufactured by microchip will be used in our project to cater the need of software and hardware.

The PIC embedded controller contains ADC, DAC, PWM and much more built-in options are there to have a better design.

In the existing we are only bothered about the mechanical efficiency which can be calculated using the torque and speed values. But in the proposed system we are dealing with the electrical and mechanical efficiency. And the efficiency is measured in more secured manner, in other terms the safety efficiency.

In the system proposed, the efficiency is measured using an absolute technique. This is also known as energy auditing. The following features will be given in our project. 


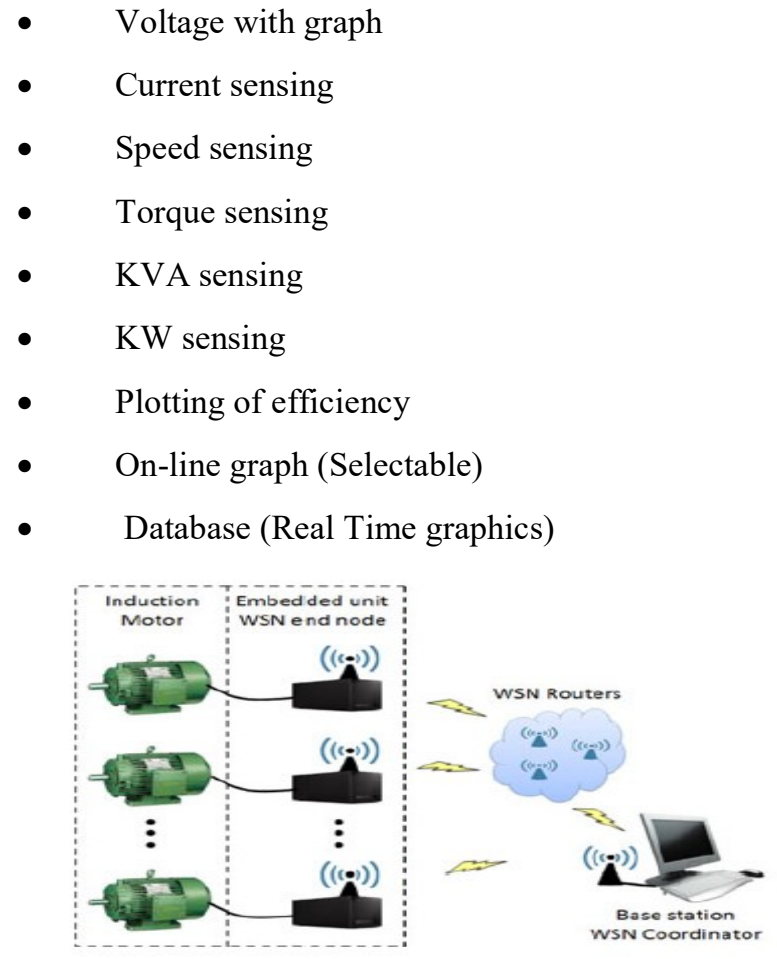

Figure 1 Embedded system integrated into the WSN

\section{SOFTWARE DESCRIPTION}

\section{A. MPLABIDE}

A development system for embedded controllers is a system of programs running on a desktop PC to help write, edit, debug and program code the intelligence of embedded systems applications into a microcontroller. MPLAB IDE, runs on a PC and contains all the components needed to design and deploy embedded systems applications.

There are some typical tasks for developing an Embeddeed Controller application, they are described as follows

- $\quad$ Create a high level design, from the specification and requirements

- Compile,assemble and link the software using assembler and compiler to convert the code to ones and zeros.

- Test the code for errors and correct them by giving notification

- $\quad$ Burn the code into the PIC microcontroller and verify that it executes correctly in the finished application.

The main components in the MPLAB IDE software are the Project Manager, Editor, Assembler/Linker \& language tools, Debugger and execution engines.

First using this software we are writing the code in Embedded $\mathrm{C}$ language and then dump it into the PIC using another software.

\section{B. $\quad$ PIC Programmer}

The PIC programmer is software that is used to dump the hex file in to the PIC controller. The PIC programmer is capable of programming a variety of Flash based Microcontrollers. It has a separate programmer/debugger unit which plug into the board carrying the chip to be programmed this makes it possible to use the programmer with a custom circuit board.

\section{Visual Basics}

Visual Basic is a tool that allows you to develop Windows (Graphic User Interface - GUI) applications. The applications have a familiar appearance to the user. Visual Basic is event-driven, meaning code remains idle until called upon to respond to some event (button pressing, menu selection). Visual Basic is governed by an event processor. Nothing happens until an event is detected. Once an event is detected, the code corresponding to that event (event procedure) is executed. Program control is then returned to the event processor.

\section{HARDWARE DESCRIPTION}

The system consist of 5 modules like Data Acquiring module, Data Processing module, Data converting module, communication module and Data manipulation module.

The rating of the $\mathrm{PT}$ used is $250 \mathrm{~V} / 6 \mathrm{~V}$, and the output is given to the voltage sensing circuit, which is a dual Op Amp (IC 1458) circuit, it consist of an integrator which will work as an signal conditioner. The out put from the circuit is given to the inbuilt ADC of the PIC where it is converted to digital values. In the current sensing part the output from the current transformer is shunted by a resistor and the value is converted to voltage, this conversion is done because, direct measurement of current is not possible. It is then given to the voltage sensing circuit. And output is given to the ADC of the PIC.

Shear beam load cell is used for the measurement of the torque. When a force is applied on the beam it will deform and the deformation is converted to the voltage signal. The used load cell is capable of detecting a weight of $10 \mathrm{Kg}$, which will give a $200 \mathrm{mV}$ value, so an amplifier, signal conditioner and sensing circuit is used for the measurement of the value. Thermistor is used for the sensing of the temperature change. The semiconductor used as the thermistor is having a negative temperature coefficient of resistance, so the change in the resistance along with the temperature change measured using a potential divider. LDR (Light Dependent Resistors) are used for the detection $f$ the presence of spark. The power factor of the motor is measured using the average and peak values of the voltage. Tachogenerator is used for the measurement of the speed. 
These values are then converted to digital values using data converting module. It is then given to the system after converting its logic family using MAX 232 IC. A voltage doubler circuit is given to change the voltage value of the data.

In the receiver side it is given to the personal computer. The GUI used is visual basic, which act as data manipulator. There it is again converted to the actual value

The proposed system mainly focuses on the need of more non-invasive monitoring of the motor. Therefore the calculation is done from another base station so that the machine interference with the working motor is smaller compared to other techniques. That is the sensors are connected with the motor in the working station, and the GUI screen and the calculation part is done at a base station. Using this technique we can reduce the inconvenience of the user to deal with the working motor and its surrounding.

The transmitter side hardware is connected with the motor. It mainly consists of different sensors and the pic microcontroller and also the wireless transmission module. The sensors used are voltage sensor, current sensor, speed sensor, torque sensor and the power factor sensor.

Like any electric motor, a 3-phase induction motor has a stator and rotor. The stator carries a 3-phase winding (called stator winding) while the rotor carries a short circuited winding (called rotor winding winding). Only the stator winding is fed from 3-phase supply. The rotor winding derives its voltage and power from the externally energized stator winding through electromagnetic induction and hence the name. the induction motor may be considered to be a transformer with rotating secondary and it can, therefore, be described as a "transformer type" ac- machine in which electrical energy is converted into mechanical energy.

Current transformers are used so that ammeters and the current coils of other instruments and relays need not be connected directly to high voltage lines. In other words, these instruments and relays are insulated from high voltages. CT's also step down the current in a known ratio. The use of CT means that relatively small and accurate instruments, relays and control devices of standardized design can be used in circuits.

The noises are filtered using Noise filter. And the analog value of the current is converted into digital by full wave precision rectifier. Filter circuits which are usually capacitors acting as a surge arrester always follow the rectifier unit. This capacitor is also called as a decoupling capacitor or a bypassing capacitor, is used not only to short the ripple with frequency of $120 \mathrm{~Hz}$ to ground but also to leave the frequency of the DC to appear at the output.

The precision rectifier, which is also known as a super diode, is a configuration obtained with an operational amplifier in order to have a circuit behaving like an ideal diode and rectifier. It can be useful for high-precision signal processing. The full-wave rectifier depends on the fact that bothe the half-wave rectifier and the summing amplifier are precision circuits. It operates by producing an inverted half wave rectified signal and then adding that signal at double amplitude to the original signal in the summing amplifier. The result is reversal of the selected polarity of the input signal.

The potential transformer operates on the same principle as a power or distribution transformer. The main difference is that the capacity of a potential transformer has rating from 100 to 500 volt amperes (VA). The low voltage side is usually wound for $115 \mathrm{~V}$. The load on the low voltage side usually consists of not only the potential coils of various instruments but may also include the potential coil of relays and other control equipments. In general the load is relatively light and is not necessary to have PT's with a capacity greater than 100 to $500 \mathrm{VA}$.

Potential divider is a linear circuit that produces an output voltage that is a fraction of its input voltage. Voltage division refers to the partitioning of a voltage among the components of the divider. An example of a voltage divider consists of two resistors in series on a potentiometer. It is commonly used to create a low voltage signal proportional to the voltage to be measured, and may also be used as a signal attenuator at low frequencies.

Signal conditioners are designed to isolate, transmit, convert, and amplify analog singals in harsh industrial environment in order to improve the reliability of the process. The signal conditioners save panel space, solve mismatched signal issues, standardize on a signal type, reduce wiring with loop powered units, and improve troubleshooting. Or it simply means manipulating an analog signal in such a way that it meets the requirements of the next stage process.

The tachogenerator gives a continuous voltage sign corresponding to the speed actual value of the electric machine to which it is coupled The Tachogenerator is fitted with permanent magnets in the stator with the function of creating a magnetic field. An amplitude continuous voltage proportional to the speed, which depends on the rotation direction, is generated in the wound rotor.

Tachogenerators are frequently used to power tachometers to measure the speeds of electric motors engines, and the equipment they power. Tachogenerators generate voltage roughly proportional to the shaft speed.

Frequency-to-Voltage converts are commonly associated with digital tachometers, which are used to measure the rate of rotation for a wheel. Digital tachometers are currently available in a wide range of forms (all of which use some type of frequency to voltage converter). Most non-contact tachometers 
require reflective strips to be placed on the object to be measured.

The frequency to voltage converter can be used for any application that requires measurement of a repeating event, such as a wheel making a complete rotation. For the robot, this could be used in place of the current encoders to measure velocity.

Theory of Operation: A rate meter simply measures the rate at which some event occurs. Usually this is done by counting the events (contact closures, electrical pulses, etc.) for a given period of time (known as the integration interval) and then simply dividing the number of events by the time to get a rate. Conversely, an analog tachometer is usually built up out of an electro-mechanical current meter and some sort of input conditioning circuitry. Instead of integrating the input signal over discrete time intervals, the mass of the meter movement itself is used to continuously integrate the input pulses.

A load cell is a transducer that is used to convert a force into electrical signal. This conversion is indirect and happens in two stages. Through a mechanical arrangement, the force being sensed deforms a strain gauge.

The strain gauge measures the deformation as an electrical signal, because the strain changes the effective electrical resistances of the wire. A load cell usually consists of four strain gauges in a Wheatstones bridge configuration. Load cells of one strain gauge (quarter bridge) or two strain gauges (half bridge) are also available. The electrical output is typically in the order of a few millivolts and requires amplification by an instrumentation amplifier before it can be used. The output of the transducer can be scaled to calculate the force applied to the transducer.

Relays are electromagnetic switches, which provides contact between two mechanical elements. There are several electrical equipments and machines of various ratings. Each needs certain adequate protection. The protective relaying senses the abnormal conditions in a part of the power system and isolates that part from the healthy part of system. The relays used in this project are compact, self-contained devices which respond to abnormal conditions (relays can distinguish normal and abnormal conditions).

PICs are commonly used in a host of different applications mainly due to their low cost and flexibility. Costing upwards of $€ 1$ they allow us to automate many of the repetitive processes which would otherwise require expensive labour. An automated welder or a traffic barrier control system are often found to have a PIC at their core. There are many types of PIC available for use but many are sourced and supplied by a US company called Microchi

The data collected from the motor is processed in the PIC and then transmitted to the base station. At the receiver end the data is collected by a receiver. And then the collected data is moved to the personal computer after processing through the microcontroller circuit.

The protocol used here for the wireless transmission is zigbee. ZigBee is a proprietary set of high level communication protocols designed to use small, low power digital radios based on the IEEE 802.15.4 standard for wireless personal area networking.

The technology is designed to be simpler and cheaper than other WPANs such as Bluetooth. The most capable ZigBee node type is said to require only about $10 \%$ of the software of a typical Bluetooth or Wireless Internet node, while the simplest nodes are about $2 \%$. As of 2004, the estimated cost of the radio for a ZigBee node is about $\$ 6$ to the manufacturer.

ZigBee is aimed at applications with low data rates and low power consumption. ZigBee's current focus is to define a general-purpose, inexpensive selforganizing mesh network that can be shared by industrial controls, medical devices, smoke and intruder alarms, building automation and home automation. The network is designed to use very small amounts of power, so that individual devices might run for a year or two with a single alkaline battery. The PC will give the GUI for the system. It gives the display of the parameters measured and the graphical representations. The GUI used is Visual Basic. 6.

In computing, a Graphical User Interface (GUI) is a type of user interface that allows users to interact with electronic devices using images rather than text commands. GUIs can be used in computers, hand-held devices such as MP3 players, portable media players or gaming devices, household appliances and office equipment. A GUI represents the information and actions available to a user through graphical icons and visual indicators such as secondary notation, as opposed to text-based interfaces, typed command labels or text navigation. The actions are usually performed through direct manipulation of the graphical elements.

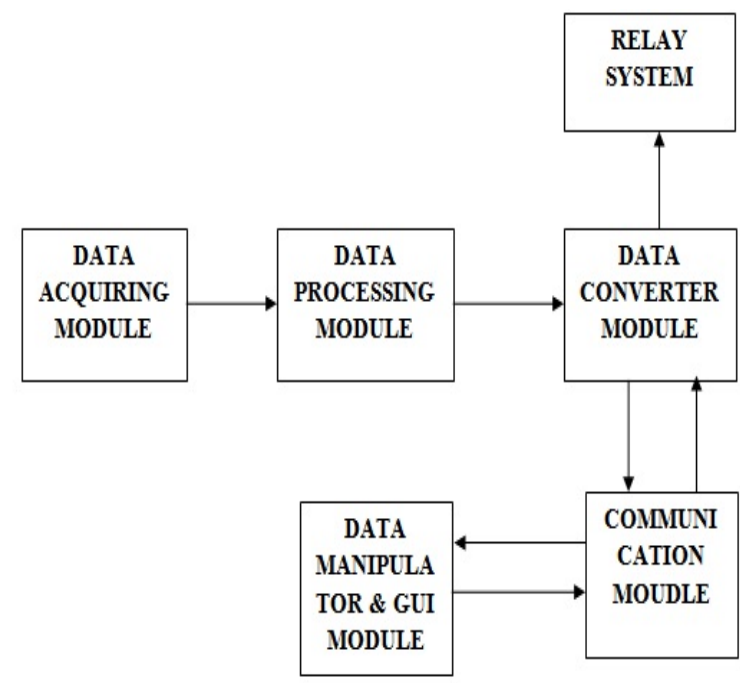

Figure 3: Modules of the system 


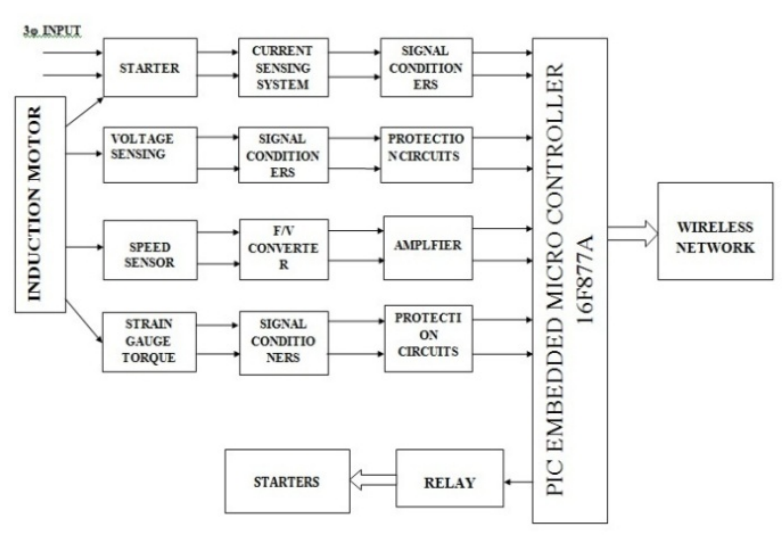

Figure 4. transmitter block diagram

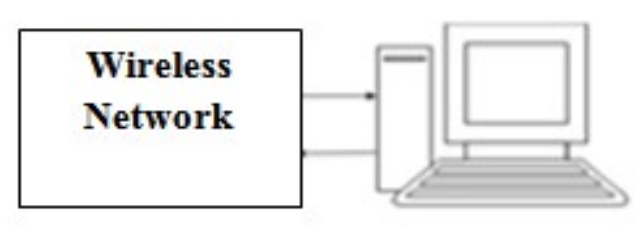

Figure 5. receiver block diagram

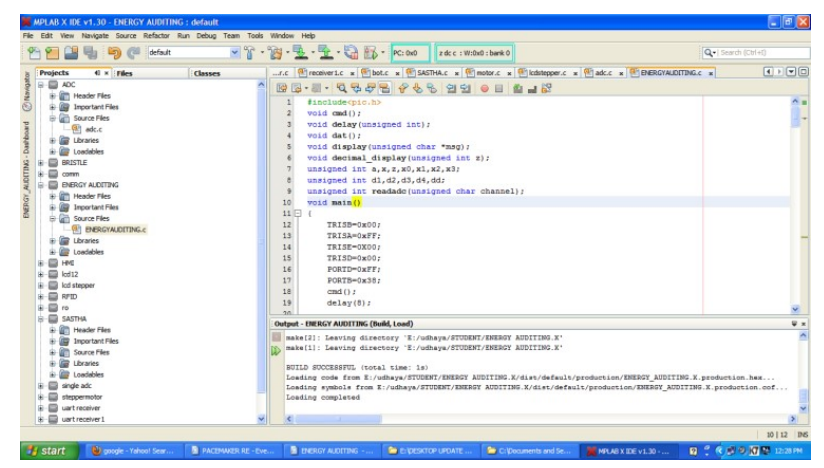

Figure 5 MPLAB IDE window

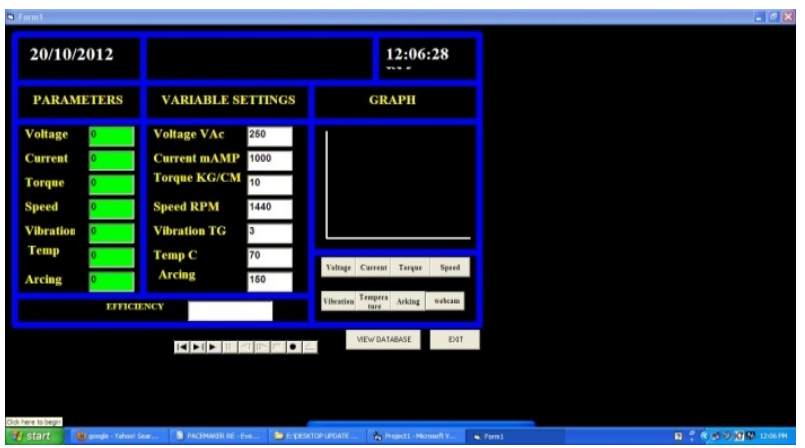

Figure 6. GUI snap shot

\section{RESULT AND DISCUSSION}

This paper presented an embedded system integrated into a WSN for online dynamic torque and efficiency monitoring in induction motors. We can use the energy auditing method to estimate shaft torque and motor efficiency. The calculations for estimating the targeted values can be done locally and then transmitted to a monitoring base unit through a WSN. The MPLAB
IDE screen shot showing the sensor input acquiring program is shown in figure 5 .

The total system can be explained as 5 modules. They are the date acquiring module, data processing module, data converting module, communication module and data manipulation module. The data acquiring module consist of the parameter sensors, and the sensing circuits. The sensors used in the circuits are Potential transformers for the sensing of voltage, Current Transformers for the sensing of current, shear beam load cell for the torque sensing and tachogenerator for the measurement of the speed of the motor. For sensing the temperature change of the system thermistor is used, and if any sparking occurs LDR will detect the light change. The sensor values are then given to PIC circuit for converting it into digital and then to MAXIC to convert its logic family and then to the Manipulator module.

Figure 6 shows the GUI window which is the manipulator module, here the data is converted to the actual values using mulitlication factors and then displayd the values and calculated efficiency. Triping command is given here.

Even with the difficulties in data transmission using the WSN in some scenarios, the system will be able to provide useful monitoring information, since all processing is done locally (i.e., only the information already computed is transmitted over the network). Without local processing, it might be impossible to use the WSN technology for this particular application, considering an unreliable transmission medium. Allied to the local processing capacity, other techniques can be developed to mitigate interference in those environments, leading to better communication performance.

\section{CONCLUSION}

The proposed energy management system in this paper is a combination of advanced techniques and plant management process, aiming for goal through technical means. It has integrated new techniques such as nonintrusive efficiency estimation.

Wireless sensor network is used to transmit datas collected from the machine to the base station. Visual basic is used for the graphical user interface. In the visual basics we calculate the efficiency of the system torque and other parameters measured.

Embedded $\mathrm{C}$ programming language is used to program the PIC microcontroller. And the software used to program is MPLAB IDE. Visual basic is the GUI for the system.

The total system gives an efficient mechanism for the measurement of the parameters of the induction motor and calculating its efficiency without interrupting the actual working of the system. 


\section{REFERENCES}

[1] B. Lu, T. G. Habetler, and R. G. Harley, "A nonintrusive and in-service motor-efficiency estimation method using air-gap torque with considerationsof condition monitoring," IEEE Trans. Ind. Appl., vol. 44, no. 6,pp. 1666-1674, Nov./Dec. 2008.

[2] J. Pedro Amaro_†, Fernando J.T.E. Ferreira,"low cost wireless sensor for in field monitoring of induction motor", ," IEEE Trans. Ind. Appl., vol. 44, no. 6,pp. 1666-1674, Nov./Dec. 2010.

[3] Yanfeng Li 1,2, Haibin Yu,"energy management of induction motors based on non-intrusive efficiency estimation", Proceeding of International Conference on Electrical Machines and Systems 2007.

[4] Nagendrappa. H1 , Prakash Bure2,"energy audit and management of induction motor using genetic algorithm" International Journal of Recent Trends in Engineering

[5] V.P. Sakthivel1 R. Bhuvaneswari2 S. Subramanian," Non-intrusive efficiency estimation method for energy auditing and management of in-service induction motor using bacterial foraging algorithm" Published in IET Electric Power Applications Received on 28th December 2009 Revised on 23rd March 2010 doi: 10.1049/iet-epa.2009.0313

[6] Abel C. Lima-Filho "Embedded system integrated into a wireless sensor network for online dynamic torque and efficiency monitoring in induction motors" Ieee/asme transactions on mechatronics, vol. 17 , no. 3 , june 2012

[7] T. Phumiphak, and C. Chat-uthai,"An Economical Method for Induction Motor Field Efficiency Estimation for Use in On-site Energy Audit and Management" 2004 Intematlonal Conference on Power System 2004

[8] R. Hanitsch, "Energy Efficient Electric Motors," in Proc. RIO 02-World Climate Energy Event, 2002, pp. 6-11.

[9] K. Kim and A. G. Parlos, "Induction motor fault diagnosis based on neuropredictors and wavelet signal processing," IEEE/ASME Trans. Mechatronics vol. 7, no. 2, pp. 201-219, Jun. 2002.

[10] A.C.Lima-Filho, F. A. Belo, and R. D. Gomes, "Tests prove, self-powered, wireless, pump torquemeter," OilGas J., vol. 106, no. 46, pp. 4348, 2008.

[11] R. B. Reich, "Rotary transformer," U.S. Patent 4 412 198, Oct. 25, 1983.

[12] W. F. Buchele, "Strain-gauge brushless torquemeter," U.S. Patent 3881 347, May 6, 1975.

[13] Z. Meng and B. Liu, "Research on torque real time monitoring system of rotary machine," Chin. J. Sci. Instrum., vol. 26, pp. 38-39, 2005.

[14] A. C. Lima-Filho, F. A. Belo, J. L. A. Santos, and E. G. Anjos, "Experimental and theoretical study of a telemetric dynamic torque meter," J. Brazilian Soc. Mech. Sci. Eng., vol. 32, pp. 241249, 2010. 